\title{
Millennial reviews
}

\section{The pathologist in the 21 st century-generalist or specialist?}

\author{
Nigel Kirkham
}

$A$ jack of all trades and master of none?

Virchow and his fellow pioneers were active in the 19th century, but pathologists are largely a creation of the 20th century and have really only been present in numbers in the second half of the century. In the United Kingdom the development of the National Health Service over this period has seen the development and expansion of hospital laboratories. This development process has been one of successive waves of specialisation.

The first consultant that I worked for had started his consultant career as a single handed general pathologist, covering all aspects of pathology in what was, for the time, quite a large district hospital. He began with a skeleton staff and by the time of his retirement was working in a laboratory that had expanded to have consultants in histopathology, haematology, and microbiology, and a senior biochemist in the chemical pathology department. In the ensuing years that laboratory has closed as a result of rationalisation and amalgamation of services. The histopathology and cytopathology services are now provided as part of the work of a larger group of consultants whose work comes from several hospitals and who are each specialised, working only in one or a small group of subspecialist areas.

The move from generalist to specialist has been far quicker in the clinical specialties, which were fewer in number 50 years ago. The general surgeon and the general physician held sway in the general hospital. The demands of increasing complexity of diagnosis and treatment combined with increasing expectations from patients and their relatives have led to considerable changes, some driven by technological advances and others by new treatments or by different approaches to the patient.

Perhaps the most important technological changes we have seen in the last 25 years have been the introduction and improvement of the flexible endoscope, the biopsy needle, and the development of various forms of scanning and imaging, to the point where the boundaries between surgeon and radiologist have been blurred by the progress of the interventional radiologist. All of this has led to considerably increased workloads for the histopathologist, but as the majority of these biopsies are taken appropriately there is no ready way of reducing much of the increase. ${ }^{1}$ As senior colleagues with a long career of generalist practice behind them retire, it often seems that virtually every keen new young consultant arriving on the scene, irrespective of specialty, will be scoping or biopsying their patients in one way or another, with the possible exception of the psychiatrists. With developments in neuroscience even they may not be exempt forever.

Many of these new clinicians are involved in the developing areas of health care, with screening and management of cancer high on the list. The cynic might suggest that the biggest change in recent years in the treatment of breast cancer has been the move for the surgeon to speak to the patient before performing the operation (and then for a supportive counsellor to follow the surgeon into the consulting room and explain what he has just said). Certainly pathologists spend an increasing proportion of their time in multidisciplinary meetings, where various aspects of the diagnosis are discussed as part of the clinical management process. No longer is pathology a refuge for struggling doctors with poor presentation skills, who in the past have been able to live out their professional life in the monastic isolation of the laboratory.

This more open approach to practice has led to increasing demands for diagnostic accuracy and for reports that contain full information on the type, grade, and stage of tumours, as well information on details of hormone receptor status, immunophenotype, and soon, no doubt, other more molecular data. ${ }^{2}{ }^{3}$ Here the generalist comes under criticism. For instance it has been said that in the development of minimum datasets, however minimal you make the datasets, many pathologists remain poor at achieving the standard.

The multidisciplinary meeting can play a part in auditing pathology reports ${ }^{4}$ but it works more satisfactorily if the reports are correct and contain all the requisite information. One of the largely, but not completely, unspoken pressures behind the push for specialisation is the perception that with specialisation will come higher standards of diagnosis and reporting. There is quite a body of evidence from audit 
studies that tends to support that case. ${ }^{5-8}$ However, it remains extremely difficult to achieve the perfect performance. Even in major teaching hospitals with active internal audit there remains an error rate of clinically important deficiencies of around $1.1-1.4 \%$, and errors of less importance in 3-4\%. ${ }^{9}$ More active prospective approaches such as routine review of every case by a second pathologist have been shown to identify and hence reduce the number of important errors in reports. ${ }^{1011}$ There is probably room for a change in thinking on this issue of diagnostic accuracy. At present a good deal of emphasis is placed on the individual pathologist. Many external quality assurance (EQA) schemes work on the basis of the individual taking some form of test as a measure of performance, or even of competence to practice. Much of medical undergraduate and postgraduate education has been designed with the aim of producing individuals capable of working alone and making their own decisions. Meanwhile in the outside world there has been much more emphasis on the development of team working. The assumption that an individual can constantly achieve $100 \%$ performance and that a group of pathologists can all achieve the same high standard is without any basis in fact.

Almost any aspect of human endeavour will be shown, when measured in an appropriate way, to follow the bell shaped or standard distribution curve. In medical discussions of performance the airlines are often called into play as an example of good practice. "Look at the airlines," someone will say, "if they did not achieve a $100 \%$ performance then we would see airplanes crashing every day of the year." A closer look at the evidence shows that the airlines also follow the bell shaped curve. Most commercial flights arrive safely. Crashes are rare: so rare as to fall outside the two standard deviations of $95 \%$ confidence. So a successful landing by an aircrew is the expected result on the overwhelming majority of occasions.

It is instructive to see how the airlines achieve this performance. The evidence suggests that not only are the pilots required to possess a good deal of knowledge about the plane they are flying and to be physically fit to fly, but they are also encouraged and trained to work together in teams; the basis for this is that if the pilot and co-pilot are talking to each other and working together on the task in hand the plane is more likely to land safely.

Whether pursuing a career as a pilot or a pathologist, personal qualities of ability, memory, pattern recognition, diligence, commitment, dedication, and judgement are required. Perhaps the time has come for us to consider some of the other similarities. Maybe we need to stop pretending that we live in some pathological version of Garrison Kieller's Lake Wobegone, where "all of the children are above average" and acknowledge that we are fallible and human. That to deliver a safe and effective service we need to look at developing support systems so that diagnostic practice is fail-safe and that error trapping is a formal part of the way in which we work.
Many do this already in a formal or an informal way. One of the most practically useful developments for the pathologist in the last 25 years has been the introduction of the double headed or multiheaded microscope. With these essential tools of the trade it is now so easy to share and discuss a difficult case with a colleague. If it became the norm for all obviously difficult, tricky, or contentious cases to be shared around with colleagues in the department, then perhaps the general standard would be higher.

There remain the small proportion of difficult cases that cannot be resolved in-house. Here the NHS has signally failed either to recognise the problem or to find a solution. This is the critical interface between the generalist and the specialist. One of the most important skills of the generalist is the ability to "know when you do not know." Most pathologists have an informal network of expert specialist pathologists whom they can call upon for the difficult case. Of course the chosen expert is probably only expert in a small area and will in turn need to call for help with problem cases outside that area. One definition, after all, of an expert is "someone who knows more and more about less and less." There are few formal studies of the performance of experts, but a study of the pathology panel of the Dutch Melanoma Working Group showed that a panel of experts was able to reduce the number of equivocal and wrong diagnoses when considering a series of difficult biopsies of pigmented skin lesions. ${ }^{12}$ Experts themselves are not infallible: errors by experts are occasionally a reason for litigation. ${ }^{13}$

The case for the generalist has several strengths. ${ }^{14}$ With a specialist service it is harder to ensure cover, and the service may become less flexible. It may be more difficult to absorb increases in workload. A specialist who wants to move may also find it more difficult. There is a theoretical risk of boredom setting in, although on the other hand the specialist will have greater opportunities to develop and to undertake and publish research.

The training offered to the majority of trainees in histopathology is in general pathology. With the uncertainties of the job market it is a brave trainee who makes an early commitment to a specific subspecialty. In the United Kingdom the existing subspecialties of neuropathology and paediatric pathology have run into recruitment problems, perhaps partly because of the loss of cross fertilisation that occurs in single subspecialty departments.

To achieve a specialist service there must be a critical mass of pathologists. Numbers are imprecise, but it has been suggested that a group of at least 15 to 20 would be necessary to establish a workable system. This would only be possible in the teaching centres at present. Considerable thought has to be given to the fair distribution of work. It is no good for a minority of the specialists to be carrying the majority of the burden of work: a situation not unknown in some large departments. A very sophisticated system of weighting factors has been tried at the Massachusetts General Hospital to try to solve this problem. ${ }^{15}$ 
In the future we may see larger groups in non-teaching centres. In the meantime the smaller district hospitals have groups of consultants ranging in size from two to seven or eight. Groups of four or five are becoming more common. Single handed practice must surely no longer be a viable option. In this situation a compromise is possible. Most specimens do not require specialist expertise. There are not enough staff for full specialisation to be achieved. Each individual should be encouraged to develop one or more areas of expertise while maintaining their generalist skills. The users would often like to have "their" pathologist. It is easier for clinicians to relate to one lead pathologist in their area who takes the clinicopathological meeting and does the specialist audit. There may be colleagues in neighbouring hospitals who offer expertise not available locally. With some careful thought, agreement, and compromise, it should be possible to find a system that works locally to balance the competing calls of ever increasing general workload and ever increasing demands for better quality specialist services. Few solutions will be identical. "A jack of many trades and master of at least one" could be a useful aim during the forthcoming period of change and realignment.

Such is the view at the beginning of the new millennium. Of one thing we can be sure. There will be many unforseen changes and forces at play in the future, which are most likely to change the way we work; at the present time we do not know what they are. ${ }^{16}$

1 Stephenson PM, Gallagher PJ. Prospective audit of mucosal biopsy specimens of the gastrointestinal tract. $\mathcal{F}$ Clin Pathol 1995;48:936-2.

2 Herrington CS. Do HPV-negative cervical carcinomas exist? - Revisited. F Pathol 1999;189:1-3.

3 Walbloomers JM, Jacobs MV, Manos MM, et al. Human papillomavirus is a necessary cause of invasive cervical canpapillomavirus is a necessary cause of invasi

4 McBroom HM, Ramsay AD. The clinicopathological meeting. A means of auditing diagnostic performance. Am $\mathfrak{f}$ ing. A means of auditing
Surg Pathol 1993;17:75-80.

Surg Pathol 1993;17:75-80.
5 Ramsay AD. Locally organised medical audit in histopatholRamsay AD. Locally organised medict
ogy. F Clin Pathol 1991;44:353-7.

6 Ramsay AD. Errors in histopathology reporting: detection and avoidance. Histopathology 1999;34:481-90.

7 Ramsay AD, Gallagher PJ. Local audit of surgical pathology. 18 month's experience of peer review-based quality assessment in an English teaching hospital. Am F Surg Pathol 1992;16:476-82.

8 Wakely SL, Baxendine-Jones JA, Gallagher PJ, et al. Aberrant diagnoses by individual surgical pathologists. Am 7 Surg Pathol 1998;22:77-82.

9 Ramsay AD. Errors by locums. Histopathology departments already audit diagnostic errors. BMf 1999;313:117.

10 Safrin RE, Bark CJ. Surgical pathology signout: routine review of every case by second pathologist. Am f Surg (17:1190-2.

11 Lind AC, Bewtra C, Healy JC, et al. Prospective peer review in surgical pathology. Am ₹ Clin Pathol 1995;104:560-6.

12 Watts JC. Is there still a need for the general surgical pathologist? Am 7 Clin Pathol 1996;106(suppl 1):S74-6.

13 Black-Schaffer WS, Young RH, Harris NL. Subspecialization of surgical pathology at Massachusetts General Hospital. Am F Clin Pathol 1996;106(suppl 1):S33-42.

14 Veenhuizen KC, De Wit PE, Mooi WJ, et al. Quality assessment by expert opinion in melanoma pathology: experience of the pathology panel of the Dutch Melanoma Working Party. I Pathol 1997;182:266-72.

15 Troxel DB, Sabella JD. Problem areas in pathology practice uncovered by a review of malpractice claims. Am ₹ Surg Pathol 1994;18:821-31.

16 Mapstone NP, Quirke P. The pathologist in the $21 \mathrm{st}$ century: man or machine? In: Kirkham N, Lemoine NR, ed. Progress in pathology, vol 3. Edinburgh: Churchill Livingstone, 1997:139-51. 